\title{
ECG and Echocardiography Processing for Decision Support in Heart Failure
}

\author{
F Chiarugi $^{2}$, S Colantonio ${ }^{1}$, D Emmanouilidou $^{2}$, D Moroni $^{1}$, \\ F Perticone $^{3}$, A Sciacqua ${ }^{3}$, O Salvetti ${ }^{1}$ \\ ${ }^{1}$ Institute of Information Science and Technologies - Italian National Research Council (CNR), Pisa, \\ Italy \\ ${ }^{2}$ Institute of Computer Science (ICS), Foundation for Research and Technology Hellas (FORTH), \\ Heraklion, Crete, Greece \\ ${ }^{3}$ Department of Cardiology, University Magna Graecia, Catanzaro, Italy
}

\begin{abstract}
This paper presents an effective way to achieve a high level integration of signal and image processing methods in the general management of heart failure, by means of a Clinical Decision Support System (CDSS). In particular, significant and suitably designed image and signal processing algorithms are introduced to objectively and reliably evaluate important features that can facilitate decisional problems in collaboration with the CDSS. Architectural details of the components of the CDSS needed for the seamless integration of image and signal analysis workflows are finally discussed.
\end{abstract}

\section{Introduction}

Signal and imaging investigations are currently a basic step of the diagnostic, prognostic and follow-up processes of heart diseases. In this area, the development of computer-aided diagnosis schemes is still attracting a lot of interests and efforts. Actually, clinical interpretation of diagnostic data largely depends on the reader's subjective point of view, knowledge and experience. Hence, signal and image processing methods, able to make this interpretation reproducible and consistent, are fundamental for reducing subjectivity while increasing the accuracy in diagnosis. The ideal goal would be to integrate signal and image processing methods for supporting physicians' decision making in their clinical routine workflow. This is precisely the main aim of this paper which addresses the integration of signal and imaging investigations with the wide-ranging services provided by Clinical Decision Support Systems (CDSSs). Actually, signal and image processing methods can be logically regarded as an embedded part of the model base of the CDSS. In such a way an effective high level integration of signal and image processing methods in the general process of care is achieved.

This being the general goal, the paper treats the concrete and paradigmatic example of Heart Failure (HF), a clinical syndrome whose management requires -from the basic diagnostic workup - the intervention of several stakeholders and the exploitation of various imaging and non-imaging diagnostic resources. Actually, it was demonstrated by recent studies and experiences that accurate heart failure management programs, based on a suitable integration of inpatient and outpatient clinical procedures, might prevent and reduce hospital admissions, improving clinical status and reducing costs [1]. HF routine practice presents several aspects in which an automatic, computer-based support could have a favourable impact. A careful investigation about the needs of HF practitioners and the effective benefits assured by decision support was performed: four problems have been identified as highly beneficial of CDSS point-of-care intervention [2], that can be referred as macro domain problems and listed up as: (i) HF diagnosis, (ii) prognosis, (iii) therapy planning, and (iv) followup. Further, detailed decision problems were identified for specifying these macro domains, focusing as much as possible on the medical users' needs; explicative examples are:

- severity evaluation of heart failure

- identification of suitable pathways

- planning of adequate, patient's specific therapy

- analysis of diagnostic examinations

- early detection of patient's decompensation

An accurate analysis highlighted that the needed corpus of knowledge mainly consisted of domain knowhow. Nevertheless, the solution of some of these problems seemed still debated in the medical community, due to the lack of validated and assessed evidence. In such cases, computational models appeared the best solution for modelling the decision making, extracting knowledge directly 
from available data. In this perspective, we have suitably designed a CDSS for the management of heart failure which combines several models of reasoning.

Having the overall organization of the CDSS being reported in [3], in this paper we first motivate signal and image investigation in the heart failure domain and introduce the suite of developed algorithms in Section 2; then Section 3 discusses how such algorithms are integrated in the CDSS services by means of a dedicated formalization of the relevant diagnostic examination and of the involved computable parameters. Some of the results described here were presented at an earlier stage in [4].

\section{Methods}

During the formalization of the main decisional problems that require the CDSS intervention and, hence, listing up all the pieces of knowledge, data and information relevant for decision making, the importance of considering and interpreting ECG signals and echocardiography images was early realized. Among all the profitable applications into decision support workflows, the following can be listed up:

- automatic or semi-automatic computation of parameters relevant in the decisional problems;

- support of physicians' case-based reasoning processes;

- discovery of novel pertinent knowledge.

While the first is typical of routine workflows in relatively simple situations, the other two can be considered advanced applications that may aid physicians in facing critical cases or problems. Actually, not only the parameters extracted from signals and images examinations are significant to physicians for formulating a response but also the data themselves can be useful for having a general overlook of a patient's situation. This means that allowing clinicians to explore data can assure the availability of a lot of other pieces of information hidden in the same data. Moreover, when dealing with a difficult case, comparing the one at hand with assessed responses for other patients' situations can be really helpful. Difficult diagnoses and, most of all, prognosis assessment are examples of these situations. For such critical problems, data processing facilities can have further relevance for the discovery of novel knowledge by granting the computation of a wide range of parameters which can be explored and put in relations in order to find out new relevant patterns. Finally, from the opposite side, opportune knowledge formalization may represent advantages in personalization of diagnostic imaging and non-imaging investigations. This means that adequate conditions could be encoded within the CDSS in order to suggest which kind of parameters could be more usefully evaluated for a given patient during, for instance, an echocardiography or an ECG session.

\subsection{Signal processing methods}

ECG is one of the very basic examinations performed in the evaluation and assessment of HF. According to [5], the negative predictive value of normal ECG to exclude left ventricular systolic dysfunction exceeds $90 \%$. The most common ECG examinations are the Resting ECG and the Holter ECG. While the latter is more commonly used for the discovery of rhythm abnormalities and the computation of the Heart Rate Variability (HRV), the former is more commonly used for the morphological evaluation of the PQRST complexes.

Considering the crucial role of ECG signals and the various related examinations, it was immediately judged important to design and implement some basic, robust and scalable algorithms for ECG processing that could be immediately applied to the raw data acquired by ECG devices with different lead numbers and different acquisition periods. After some interviews with the clinicians, it has been identified a significant operative scenario, where the ECG acquired with a non-interpretive electrocardiograph is transferred to the hospital gateway and from there processed in order to:

1. Detect the QRS complexes

2. Identify the dominant beats

3. Evaluate the averaged dominant beat (for all the leads)

In particular, the averaged dominant beat can be used by the cardiologists (with the help of a graphical ECG viewer), for the evaluation of all the measurements of interest for the diagnosis or the follow-up of heart failure patients, like ST depression, QRS and QT durations, Sokolow-Lyon index for left ventricular hypertrophy, presence of left or right branch bundle block and presence of pathological Q waves. Notice that, since the average dominant beat is cleaner from the noise than the original signal, performing measurements on this average beat leads to a more accurate results, thus reducing inter- and intra- observer variability. The details for the developed algorithm for QRS detection and its performance may be found in $[4,6]$. The two tasks corresponding to the identification of dominant beats and the construction of the averaged dominant beat are solved by the algorithms described in [7].

\subsection{Image processing methods}

Imaging techniques offer invaluable aid in the objective documentation of cardiac function, allowing for the computation of parameters relative to chamber dimensions, wall thickness, systolic and diastolic function, regurgitations and pulmonary blood pressure. Chest X-ray and echocardiography should be included in the HF initial diagnostic workup [5]. Further, echocardiography will be regularly repeated to monitor in an objective way the changes in the clinical course of a HF patient. Addi- 
tional techniques, like nuclear imaging and cardiac magnetic resonance, may be also considered for particular patients, since they have not been shown to be superior to echocardiography in the management of most HF population. Thus, echocardiography and in particular 2-D TransThoracic Echocardiography (TTE) for its portability and versatility is the key imaging technique for the practical management of HF. The most important measurement performed by TTE is Left Ventricle Ejection Fraction (LVEF), which permits to distinguish patients with cardiac systolic dysfunction from patients with preserved systolic function. After having computed the End-Diastolic Volume (EDV) and the End-Systolic volume (ESV), the LVEF is given by:

$$
\mathrm{LVEF}=(\mathrm{EDV}-\mathrm{ESV}) / \mathrm{EDV}
$$

Among different models for the computation of left ventricular volumes, the American Society of Echocardiography [8] suggests the use of the so-called Simpson's rule, by which the left ventricle is approximated by a stack of circular (or elliptical) disks. Simpson's method, therefore, relies on left ventricle border tracing. It is well-known that manual border tracing, besides being timeconsuming, is prone to inter- and intra- observer variability, and thus is unable to provide a satisfactory and reproducible measurement of LVEF. Image processing techniques may reduce variability of human interventions in border tracing, by providing automated or, at least, semiautomated methods for tracing contours of relevant structures found in an image. However, the segmentation problem for ultrasound images is by no means trivial, due mainly to low signalto-noise ratio, low contrast, image anisotropy and speckle noise [9]. From these considerations, it was clearly realized that the development of assisted segmentation methods, able to deal with echocardiographic image sequences, could represent a valid support to the physicians in the process of image report formation. Thus, a prototypical toolkit -composed of three main modules- was developed for the analysis of apical-view sequences of the heart. The first module (Region Identification), which takes in input an apical sequence of the heart, is able to identify the left ventricle cavity in every frame of the sequence by means of mimetic criteria, providing a rough segmentation. The second module (Segmentation Refinement), which takes in input an image and a rough segmentation of it, is able to refine the segmentation exploiting a variational formulation of level set methods, which achieves regularization of the boundary of the left ventricle as well as better adherence to image edges [10]. The result of level set segmentation in the end-systole and end-diastole frames is shown in Figure 1. The third module (Feature Extraction) is able to extract significant features from a set of segmented left ventricles, the most important being EDV and ESV (both computed according to Simpson's rule) and, in turn, LVEF.

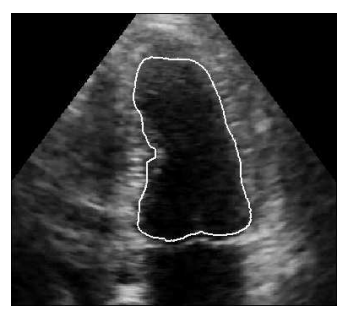

(a)

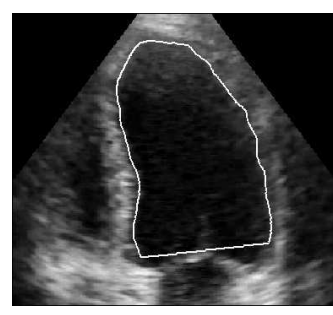

(b)
Figure 1. Final result of segmentation in an end-systole (a) and in an end-diastole (b) frame

The proposed image processing toolkit could be easily extended in several ways. Besides integrating standard tools for performing graphically image measurements (such as linear measurements) and producing IHEcompliant Simple Image and Numeric Reports, the core segmentation modules may be adapted to deal with other echocardiographic views, so as to perform a complete quantification of heart chambers.

\section{Architectural details and results}

The intervention of signal and image processing methods into the management of care delivery, as detailed in the previous sections, has been carefully and deeply investigated while designing the CDSS, identifying its functionalities and modeling its architecture. The CDSS has been devised for processing patients' related information by exploiting the relevant medical knowledge which has been opportunely elicited from medical experts and extracted from clinical guidelines. The symbolic paradigm has been selected for formalizing such knowledge into an ontology- and rule-based Knowledge Base (KB) [2]. During the knowledge representation process, the integration of both signal and image processing methods has been conceived in order to embody parameters extracted from different data acquisition modalities into the more general process of health care management. In particular, the integration has been focused on two main issues, i.e. (i) supplying relevant parameters to the inferential processes and (ii) personalizing the diagnostic investigations by suggesting which parameters should be extracted. An example can be used for better explaining the implications of these two issues: while processing a patient's information for identifying the causes of his worsening, the CDSS may need a number of routine parameters not yet available. In such a case, a suggestion will be issued by the system asking the clinician to perform additional examinations, such as an ECG or a TTE, in order to obtain the missing parameters. On the other side, it can happen that such routine parameters are not able to completely explain patient's status and thus the system can require the extraction 
of other non standard features that can enlighten patient's peculiar conditions. In both cases, the inferential process pauses, waiting for additional information. Reactivating the inferential process requires data processing algorithms to be performed. The CDSS has been hence carefully and specifically designed for incorporating this kind of functioning. A multilevel conceptualization strategy, which distinguishes between the knowledge and processing components, has been used so as to provide an implementationindependent description of the role that various knowledge elements play during the decision supporting process.

In brief, the CDSS is composed by (i) the Domain $K B$ which maintains the domain knowledge, formalized from the guidelines and from the clinicians' know-how; (ii) the Model Base which contains the computational decision models, signals and images processing methods and pattern searching procedures; (iii) the Meta $K B$ which is composed by the strategy knowledge about the organization of the CDSS tasks; (iv) the Brain which is the system component endowed with the reasoning capability. In particular, the brain is divided into a meta level composed by a Strategy Controller, that manages and orchestrates the object level according to the Meta $\mathrm{KB}$, and an object level containing both an Inference Engine for reasoning on the Domain KB and a Model Manager for handling computational reasoning.

The integration of signal and image processing models are, first of all, assured by a dedicated formalization of the relevant acquisition modalities, diagnostic examinations and computable parameters within the ontologies of the Domain KB. Plus, inferential rules able to process parameters extracted from both signals and images are encoded into the same KB. Finally, the Meta KB contains suitable procedural rules for integrating the application of the data processing methods into the inferential reasoning process. More precisely, when the Inference Engine stops into a crisis status due to the missing values of specific parameters, the Strategy Controller is able to solve the problem by requiring to the application the opportune processing methods triggered by the Model Manager.

\section{Discussion and conclusions}

In this paper we have presented a suite of diagnostic signal and image processing algorithms and we have described their integration into the wide-ranging services provided by a CDSS for the management of heart failure. The integration is assured by a dedicated formalization of the relevant acquisition modalities, diagnostic examinations and computable parameters within an ontology of the Domain KB. Inferential rules able to process parameters extracted from both signals and images were encoded into the same KB. Future activities will focus on the extension of the already developed signal and image processing suite as well as on the realization of an integrated interface for its easy usage in conjunction with the CDSS.

\section{Acknowledgements}

This work was partially supported by European Project HEARTFAID “A knowledge based platform of services for supporting medical-clinical management of the heart failure within the elderly population"(IST-2005-027107).

\section{References}

[1] Perlini S et al. Treatment of chronic heart failure: an expert system advisor for general practitioners. Acta Cardiol. 1990;45(5):365-78.

[2] Colantonio $S$ et al. Decision support and image \& signal analysis in heart failure. A comprehensive use case. Proc. of the First International Conference on Health Informatics, HEALTHINF 2008. Funchal, Madeira, Portugal: 28-31 January $2008 ; 288-295$.

[3] Colantonio S, Martinelli M, Moroni D, Salvetti O, Perticone F, Sciacqua A, Gualtieri A. An approach to decision support in heart failure. In Proc. Semantic Web Applications and Perspectives (SWAP 2007). Bari, Italy, 18-20 December 2007.

[4] Chiarugi F, Colantonio S, Emmanouilidou D, Moroni D, Salvetti $\mathrm{O}$. Biomedical signal and image processing for decision support in heart failure. In Advances in Mass Data Analysis of Images and Signals, MDA 2008, Leipzig, Germany, July 14,$2008 ; 38-51$.

[5] Swedberg K et al. The Task Force for the diagnosis and treatment of CHF of the European Society of Cardiology, Guidelines for the diagnosis and treatment of Chronic Heart Failure. European Heart Journal 2005;45 pages.

[6] Chiarugi F, Sakkalis V, Emmanouilidou D, Krontiris T, Varanini M, Tollis I. Adaptive threshold QRS detector with best channel selection based on a noise rating system. In Proc. of Computers in Cardiology, volume 34. 2007; 157160.

[7] Chiarugi F, Emmanouilidou D, Tsamardinos I, Tollis I. Morphological classification of heartbeats using similarity features and a two-phase decision tree. In Proc. of Computers in Cardiology. 2008; Accepted for publication.

[8] R.M. Lang et al. Recommendation for Chamber Quantification. J Am Soc Echocardiogr 2005; 18:1440-1463.

[9] Noble J, Boukerroui D. Ultrasound image segmentation: a survey. IEEE Trans Med Imag 2006;25:987-1010.

[10] Barcaro U, Moroni D, Salvetti O. Automatic computation of left ventricle ejection fraction from dynamic ultrasound images. Pattern Recognition and Image Analysis 2008; 18(2):351-358

Address for correspondence:

Ovidio Salvetti

Via Moruzzi 1, 56123, Pisa (Pi), Italy

ovidio.salvetti@isti.cnr.it 